\title{
Ultrashort Optical Pulse Transmission Characteristics of Vertically Coupled Microring Resonator Add/Drop Filter
}

Shuichi Suzuki, Yasuo Kokubun, Member, IEEE, Member, OSA, Masataka Nakazawa, Fellow, IEEE, Fellow, OSA, Takashi Yamamoto, and Sai Tak Chu, Member, IEEE

\begin{abstract}
The ultrashort optical pulse transmission characteristics of a vertically coupled microring resonator (VCMRR) filter were measured at $1.55 \mu \mathrm{m}$ wavelength, and the transmission capacity per channel was evaluated. The pulsewidth at the output was measured to be $3.4 \mathrm{ps,}$, when $1.2 \mathrm{ps}$ input pulse with a sech pulse shape from a fiber laser was incident on the microring resonator filter with a fullwidth at half-maximum (FWHM) of $0.75 \mathrm{~nm}(94$ GHz). From this result, the maximum transmission capacity of the filter was estimated to be approximately $50 \mathrm{~Gb} / \mathrm{s}$. The pulse broadening through the filter was theoretically analyzed, and it was confirmed that the pulse broadening evaluated from the bandwidth of the filter spectrum response were in good agreement with the measured results.
\end{abstract}

Index Terms-Add/drop filter, microring resonator, optical waveguide device, transmission capacity, transmission characteristics.

\section{INTRODUCTION}

$\mathbf{M}$ ICRORING resonator devices are attractive as add/drop wavelength filters for wavelength multiplexed access networks, due to their functionality [1], [2] and compactness [3]. The ultracompact ring resonator can be realized by the high index contrast waveguide consisting of glass core and air cladding and the vertically coupled configuration [4], [5], where a microring resonator with a radius of a few tens of microns is stacked at the crossing point of cross-grid bus waveguides. Due to the stacked configuration, the upper and lower waveguides play different roles, i.e., the lower buried channel waveguides serve as input/output bus guides while the ring functions as the frequency selective element. In addition, the coupling strength between the ring and bus waveguides can be controlled more precisely than the lateral coupling, because the vertical separation is obtained by precisely controlling the deposition, rather than etching fine gaps. This allows us the fabrication of high $Q$ micro-ring resonator filters with a

Manuscript received June 1, 2000; revised October 11, 2000. This work was supported in part by a Grant-in-Aid for Scientific Research (B) 10555015 and (A) 11305028 of the Japan Society for the Promotion of Science, Support Center for Advanced Telecommunications Technology Research, and the International Communications Foundation.

S. Suzuki and Y. Kokubun are with the Faculty of Engineering, Department of Electronic and Computer Engineering, Yokohama National University, Yokohama 240-8501, Japan (e-mail: kokubun@dnj.ynu.ac.jp).

M. Nakazawa and T. Yamamoto are with NTT Network Innovation Laboratory, Yokosuka 239-0847, Japan.

S. T. Chu is with Little Optics Inc., Greenbelt, MD 20770 USA

Publisher Item Identifier S 0733-8724(01)01092-1.

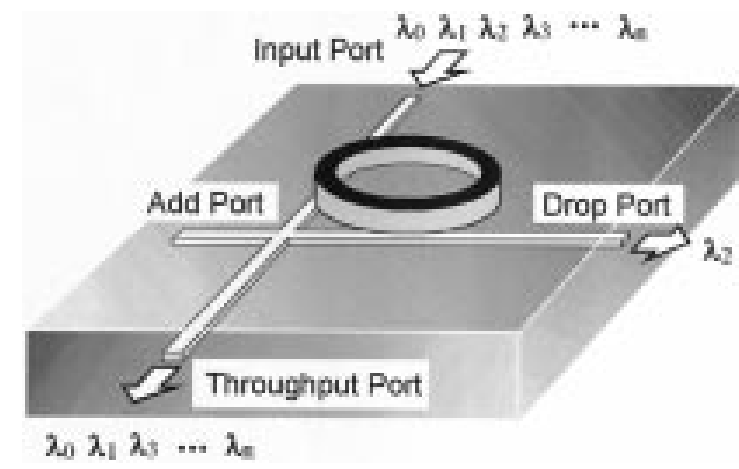

Fig. 1. Vertically coupled microring resonator add/drop filter.

very small radius of $10-20 \mu \mathrm{m}$, which exhibit bandwidths of $0.2-1.0 \mathrm{~nm}$ and a free spectral range (FSR) of $10-25 \mathrm{~nm}$.

We have proposed and demonstrated a vertically coupled microring resonator (VCMRR) filter [5]-[10] as an add/drop filter for wavelength division multiplexed access networks. However, although the generalized dispersive properties of optical filters are analytically calculated [11], [12], there has been no detailed experimental or theoretical evaluation about the relationship between the bandwidth of the filter spectrum response and the maximum transmission capacity. In this study we measured the temporal transmission characteristics of a VCMRR filter using an ultrashort pulse from a fiber laser and evaluated the transmission capacity per channel. The relationship between the bandwidth of the filter and the output pulsewidth was analyzed using the Fourier transforms (FTs), and the theoretical results were compared with the experimental data.

\section{Vertically COUPLED Microring Resonator AdD/DroP FILTER}

Fig. 1 shows the structure of the VCMRR filter. It consists of an input bus waveguide and an add/drop waveguide which are crossed at right angles. At the node, a ring waveguide, which serves as the wavelength selective crossconnect between the input and the corresponding add/drop waveguide, is placed on the crossed waveguides. To apply the ring resonator filter for WDM systems, a ring radius of a few micrometers is needed because the FSR of the filter is required to be a few tens of nanometers. Thus, the waveguide used for the VCMRR filter that we have proposed requires a high index contrast to reduce the bending loss caused by the small radius. In addition, the 


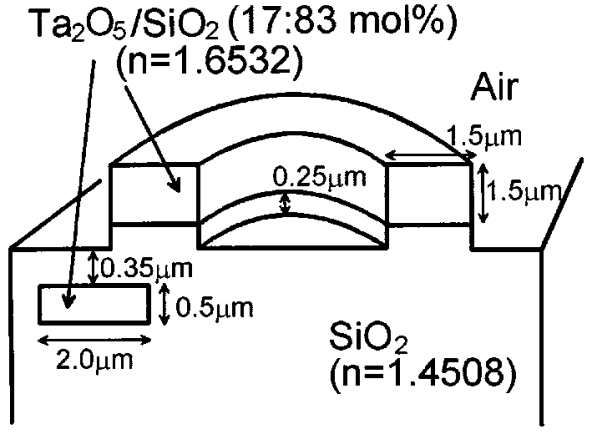

Fig. 2. Cross section of VCMRR filter used in the measurement.

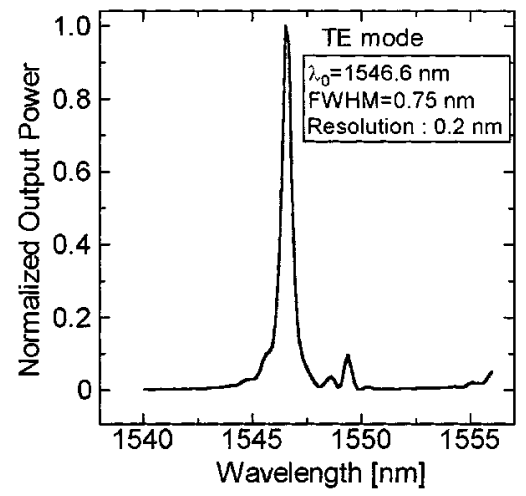

(a)

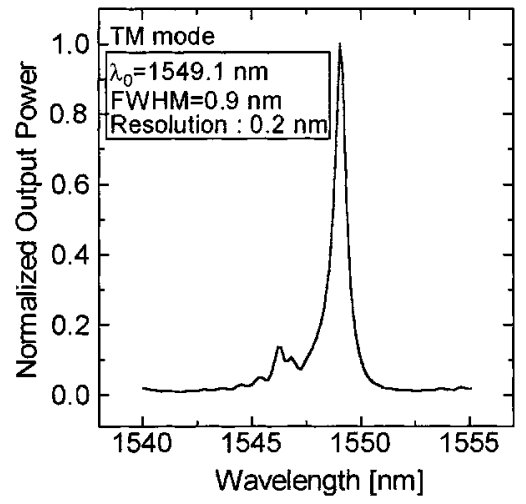

(b)

Fig. 3. Spectrum response of VCMRR filter: (a) TE polarization and (b) TM polarization.

bus waveguide layer and the ring resonator layer can be optimized independently owing to the vertically coupled configuration. Fig. 2 shows the cross-sectional structure of the VCMRR filter used in the measurement. In this device, the ring and bus waveguide cores were formed of a compound glass with a composition of $17 \mathrm{~mol} \%$ of $\mathrm{Ta}_{2} \mathrm{O}_{5}$ and $83 \mathrm{~mol} \% \mathrm{SiO}_{2}$, giving a refractive index of 1.6532 at $1.55 \mu \mathrm{m}$ wavelength. The cladding and buffer layers were made of $\mathrm{SiO}_{2}$. The width and thickness of the bus waveguide are $2.0 \mu \mathrm{m}$ and $0.5 \mu \mathrm{m}$, respectively, and those of the ring waveguide are $1.5 \mu \mathrm{m}$ and $1.5 \mu \mathrm{m}$, respectively. The radius of the ring waveguide is $20 \mu \mathrm{m}$. Details of the fabrication process were reported in [5].

The spectrum responses of the filter measured using a tunable LD are shown in Fig. 3(a) (TE polarization) and Fig. 3(b) (TM polarization). The spectrum response of the VCMRR filter

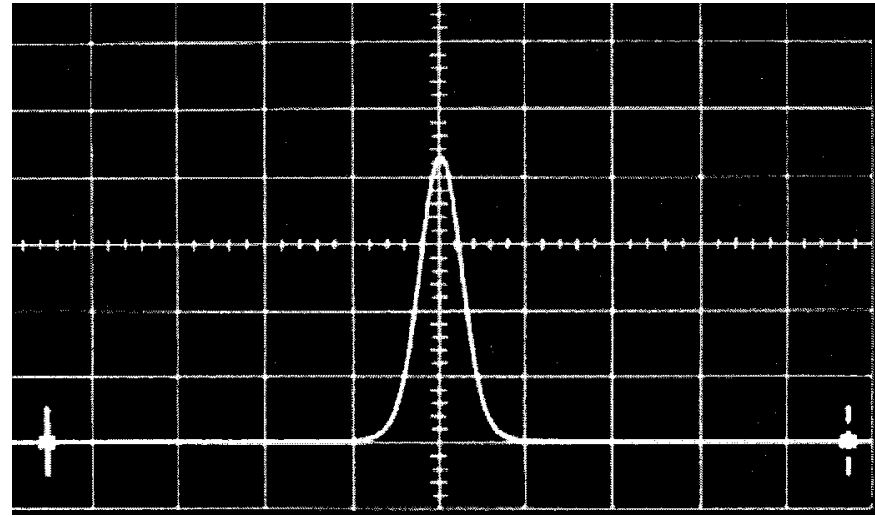

Fig. 4. Autocorrelation measurement of input pulse (3.19 ps/div).

is approximately expressed by a Lorentzian function, and the FWHM bandwidth for TE polarization was $0.75 \mathrm{~nm}(94 \mathrm{GHz})$ and that for TM polarization was $0.9 \mathrm{~nm}(112 \mathrm{GHz})$. This difference of spectral widths between TE and TM polarizations is caused by the difference of confinement factor between two polarizations.

Other polarization problems are the polarization dependence of resonant wavelength, PDL and PMD. The polarization dependence of resonant wavelength as seen in the [6] can be eliminated by a new polarization independent microring structure using the birefringent material as the overcladding, and this will be discussed in future. The difference of spectral width for TE and TM polarizations will also be improved by the polarization independent microring resonator. The PDL is small $(<1 \mathrm{~dB})$ as shown in [6] and will be reduced further in the polarization independent device. The PMD, which is defined by the difference of pulse arrival time for TE and TM polarizations, will not occur in this device, because the optical path length is very short compared with the optical fiber.

\section{Ultrashort Optical Pulse Transmission CHARACTERISTICS OF FILTER}

An ultrashort pulse train from a regeneratively modelocked fiber laser [13] with a $\operatorname{sech}(a t)$ pulse shape ( $a$ is a constant) was incident on the filter. The input pulse was linearly polarized, and the direction of polarization was controlled to be TE or TM polarization using a polarization maintaining fiber and a fiber rotation manipulator. The input pulse shape observed with an autocorrelator is shown in Fig. 4. The pulsewidth and the FWHM bandwidth of the input pulse are 1.2 ps and $2.1 \mathrm{~nm}$, respectively. The wavelength was adjusted to a resonant wavelength of the filter, i.e., $1546.6 \mathrm{~nm}$ for TE and $1549.1 \mathrm{~nm}$ for TM. The output pulse shapes from the filter observed with the autcorrelator are shown in Fig. 5 for TE polarization and Fig. 6 for TM polarization. When the output pulse shape is assumed to be described by a $\operatorname{sech}^{2}(a t)$ function, the actual pulsewidth is 0.65 times the autocorrelation width. This results in an output pulsewidth of $3.4 \mathrm{ps}$ for TE polarization. Similarly, the output pulsewidth for TM polarization was 2.8 ps. From these results, we can estimate the transmission capacity. As a pulse interval is typically set to be five to six times the output pulsewidth, the pulse interval in the present case is calculated to be 20 ps. This 


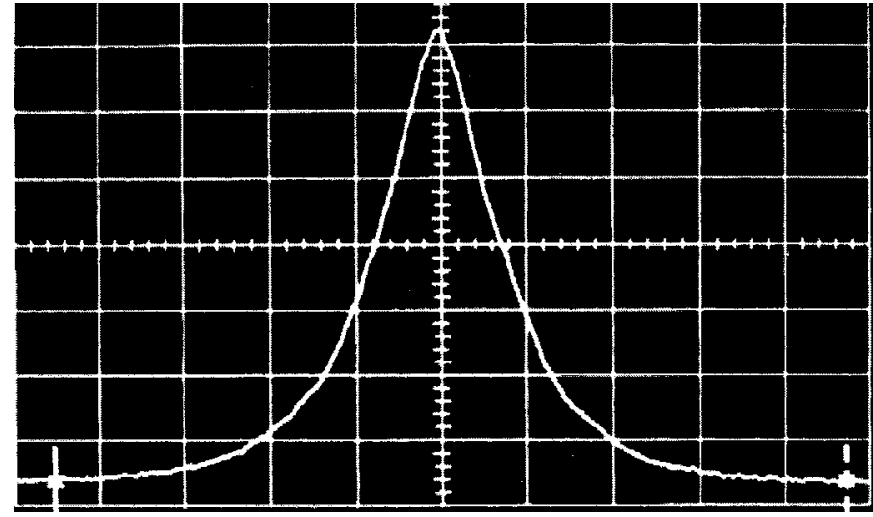

Fig. 5. Autocorrelation measurement of output pulse: TE polarization (3.19 $\mathrm{ps} / \mathrm{div})$.

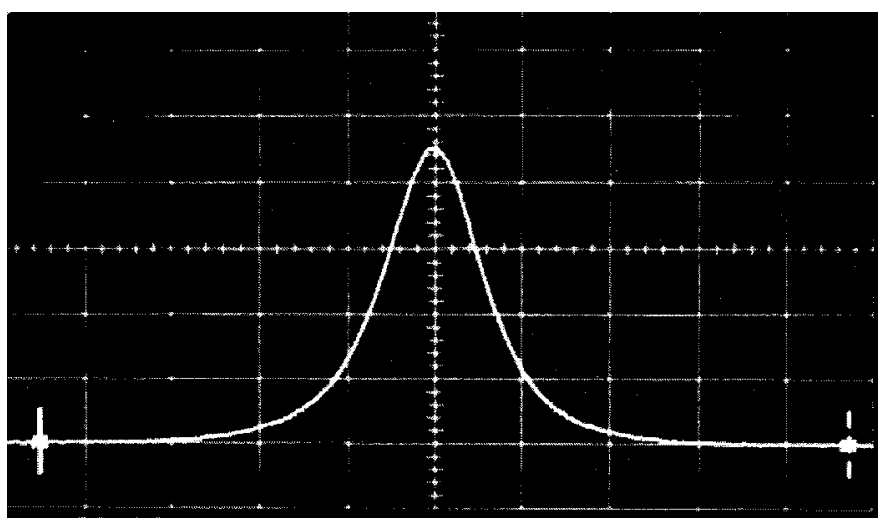

Fig. 6. Autocorrelation measurement of output pulse: TM polarization (3.19 ps/div)

corresponds to a transmission capacity per channel of $50 \mathrm{~Gb} / \mathrm{s}$. Thus this filter can be applied to $50 \mathrm{~Gb} / \mathrm{s}$ transmission systems.

Although the maximum transmission capacity was confirmed to be about $50 \mathrm{~Gb} / \mathrm{s}$, the channel spacing in the WDM system is not determined by the bandwidth of the filter but the crosstalk characteristics. Since the Lorentzian response of single microring filter has large wings and the roll-off from the resonant to off-resonant state is very slow. This will be an obstacle to the dense WDM. This problem can be improved by higher order resonators [1] consisting of two or three coupled resonators and by the cascaded resonators [7].

Next, we observed the eye pattern from the VCMRR filter as shown in Fig. 7. The light source is the same fiber laser as used above, and the repetition rate, pulsewidth, and peak power are 10 $\mathrm{Gb} / \mathrm{s}, 1.2 \mathrm{ps}$, and about $0 \mathrm{dBm}$, respectively. It is seen from Fig. 7 that the eye is clearly open. The bit error rate (BER) characteristic was measured by using the measurement system shown in Fig. 8. In this system, the insertion loss of the filter was compensated for by using erbium-doped fiber amplifiers (EDFAs). The insertion loss of the filter is about $25 \mathrm{~dB}$ [4], and this loss is mainly attributed to the input/output coupling loss caused by the large spot size mismatch between the busline waveguide $(\sim 2$ $\mu \mathrm{m})$ and the fiber $(\sim 10 \mu \mathrm{m})$, which is about $18 \mathrm{~dB}$. This coupling loss to the fiber will be reduced by integrating the spot size transformer to match the spot size of input and output waveguides to that of the fiber. The measured BER characteristic is

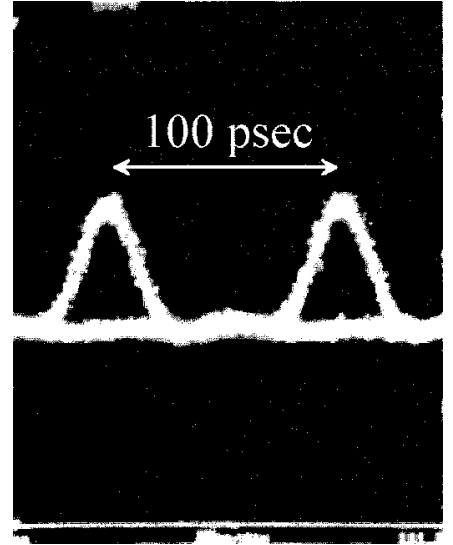

Fig. 7. Measurement result of eye pattern (after filter)

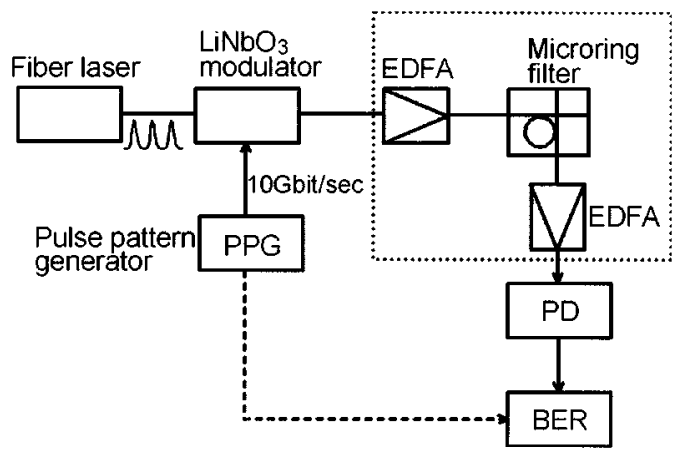

Fig. 8. Measurement system for BER characteristics.

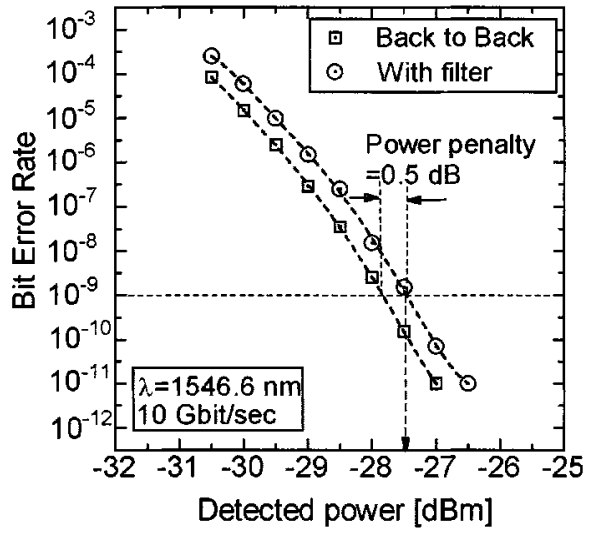

Fig. 9. BER characteristics.

shown in Fig. 9. In this figure, the back-to-back BER characteristic and the BER characteristic after the filter are shown by $\square$ and $\odot$, respectively. The power penalty was $0.5 \mathrm{~dB}$, which is mainly attributed to the SNR of the EDFAs.

\section{THEORETICAL CONSIDERATION FOR TRANSMISSION CHARACTERISTICS}

We analyzed theoretically the transmission characteristics of the VCMRR filter. First, we calculated the output pulsewidth from the filter bandwidth and the input pulsewidth by using FTs, and compared the calculated and measured results. Next, assuming that the output pulse shape was approximately expressed by an exponential or a sech-type function, the product of $\Delta T$ 
and $\Delta \nu$ was calculated. Lastly, the calculated results were compared with the values of transform limited condition of exponential and sech functions, respectively.

\section{A. Calculation Using the Fourier Transform}

The input pulse shape from the fiber laser is given by

$$
\left|f_{\text {in }}(t)\right|^{2} \propto \operatorname{sech}^{2}(a t)
$$

where $f_{\mathrm{in}}(t)$ is the electric amplitude function, and $a$ is a constant corresponding to the pulsewidth. The spectrum response of the VCMRR filter is expressed by the Lorentzian function, given by

$$
|H(\omega)|^{2} \propto \frac{1}{1+b^{2}\left(\omega-\omega_{0}\right)^{2}} .
$$

The actual response measured by a spectrum analyzer and a tunable $\mathrm{LD}$ is given by $|H(\lambda)|^{2}$ as a function of wavelength $\lambda$. The calculation procedure is as follows.

First, the value of $a$ corresponding to the input pulsewidth is calculated from the measured pulsewidth. $F_{\text {in }}(\omega)$ is the FT of $f_{\text {in }}(t)$. Next, the transfer function of the filter $H(\omega)$ is converted to $H(\lambda)$ using the relation $\omega=(2 \pi c) / \lambda$, and the value of $b$ which corresponds to the bandwidth of the spectrum response is calculated from the measured bandwidths. Finally, the FWHM of $\left|f_{\text {out }}(t)\right|^{2}$ is calculated by the inverse FT of the product $F_{\mathrm{in}}(\omega) H(\omega)$, which is given by

$$
f_{\text {out }}(t)=\frac{1}{2 \pi} \int_{-\infty}^{\infty} F_{\text {in }}(\omega) H(\omega) e^{j \omega t} d \omega .
$$

The value of the FWHM obtained above is the theoretical value of the output pulsewidth.

First, the value of $a$ in (1) is determined to be $1.47 \times 10^{12} / \mathrm{s}$ so that the input pulse expressed by (1) has a $1.2 \mathrm{ps}$ pulsewidth. The FT of $f_{\text {in }}(t)$ is given by

$$
F_{\mathrm{in}}(\omega) \propto \operatorname{sech}\left[\frac{\pi\left(\omega-\omega_{0}\right)}{2 a}\right] .
$$

Next, the transfer function $|H(\omega)|^{2}$ given by (2) is expressed in terms of wavelength $\lambda$ as

$$
|H(\omega)|^{2}=\frac{1}{1+b^{2}\left(\omega-\omega_{0}\right)^{2}}=\frac{1}{1+b^{2}\left(\frac{2 \pi c}{\lambda}-\frac{2 \pi c}{\lambda_{0}}\right)^{2}}
$$

and $b$ is determined to be $3.39 \times 10^{-12} \mathrm{~s} / \mathrm{rad}$ for TE and $2.82 \times$ $10^{-12} \mathrm{~s} / \mathrm{rad}$ for TM so that the $|H(\lambda)|^{2}$ has a FWHM corresponding to the measured bandwidths of the filter $(0.75 \mathrm{~nm}$ for TE and $0.9 \mathrm{~nm}$ for TM polarization). Substituting (4) and the following function in the angular frequency domain:

$$
H(\omega)=\frac{e^{-j \frac{\omega n_{e q} 2 \pi R}{c}}}{1+j b\left(\omega-\omega_{0}\right)}
$$

into (3), the output pulse shape $\left|f_{\text {out }}(t)\right|^{2}$ is obtained.

The calculated result is shown in Fig. 10, where the theoretical pulsewidth is expressed as a function of the bandwidth of the filter. The calculated result is in good agreement with the

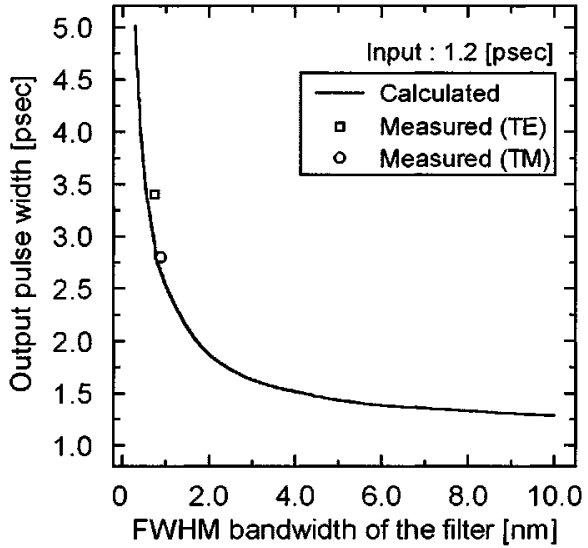

Fig. 10. Relationship between FWHM bandwidth of the filter and pulsewidth of output signal.

measured results. Thus, it can be concluded that the pulsewidth of output signal from the filter can be predicted by the FT, as long as the source wavelength is adjusted to the resonant wavelength of the filter.

\section{B. Consideration on Output Pulse Waveform}

If the input pulse with a $1.2 \mathrm{ps}$ pulsewidth ( $2.2 \mathrm{~nm}$ bandwidth) is approximated to be a $\delta$ function,

$$
\left|F_{\text {out }}(\omega)\right|^{2} \propto|H(\omega)|^{2}
$$

where $F_{\text {out }}(\omega)$ is the FT of $f_{\text {out }}(t)$, and an approximation of $\left|F_{\text {in }}(\omega)\right|^{2} \simeq$ const. is used. Thus, the output spectrum is given by the filter response which is expressed by the Lorentzian function. The impulse response corresponding to the Lorentzian spectrum is

$$
h(t) \propto\left\{\begin{array}{cc}
\exp \left(-\frac{t}{b}\right) & (t \geq 0) \\
0 & (t<0)
\end{array}\right.
$$

and the transform limited pulse expressed by (8) satisfies the following relation:

$$
\Delta T \cdot \Delta \nu \simeq 0.11
$$

where $\Delta T$ and $\Delta \nu$ are the FWHMs of the intensity of pulse and the spectrum response, respectively.

On the other hand, we also consider the case when the input spectrum $F_{\text {in }}(\omega)$ is expressed by (4). We assume that the output spectrum is approximately

$$
\begin{aligned}
\left|F_{\text {out }}(\omega)\right|^{2} & \propto \operatorname{sech}^{2}\left(\frac{\pi}{2 a}\left(\omega-\omega_{0}\right)\right) \times \frac{1}{1+b^{2}\left(\omega-\omega_{0}\right)^{2}} \\
& \cong \operatorname{sech}^{2} c\left(\omega-\omega_{0}\right)\left(c \simeq\left(\frac{\pi}{2 a}\right)^{2}+b^{2}\right)
\end{aligned}
$$

that is, the output spectrum is expressed by the sech ${ }^{2}$ function which has a narrower bandwidth than Fig. 3(a) and (b). Then the transform limited condition for the $\operatorname{sech}^{2}$ pulse is given by

$$
\Delta T \cdot \Delta \nu \simeq 0.32 .
$$

Now, we compare the measured results with the values given by (9) and (11). In this comparison, the FWHM of the spectrum 


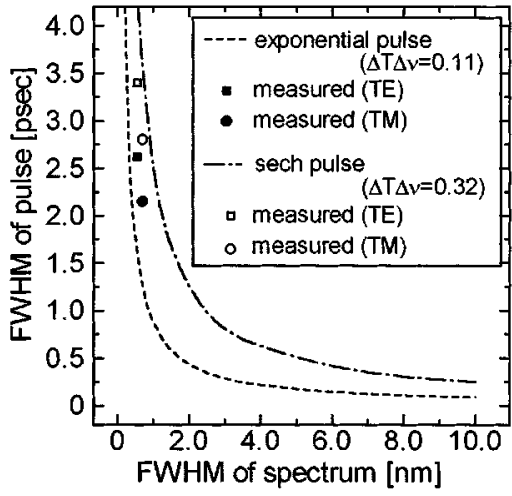

Fig. 11. Relationship between the pulsewidth and the bandwidth (transform limited pulse condition).

of the output pulse is smaller than the values shown in Fig. 3(a) and (b) which were measured using a $\mathrm{CW}$ tunable light source. This is because the spectrum responses shown in Fig. 3(a) and (b) correspond to the FT of the impulse response for which the light source has a very broad (white) spectrum bandwidth. When a sech ${ }^{2}$ pulse light source with 1.2 ps pulsewidth was incident on the filter, the FWHM of the output spectrum was $0.55 \mathrm{~nm}$ for TE polarization, and $0.71 \mathrm{~nm}$ for TM polarization. Since the pulse shape was observed with an autocorrelator, when the pulse shape is assumed to be an exponential function corresponding to a Lorentzian spectrum, $\Delta T$ is given by 0.5 times the measured value with the autocorrelator as follows:

$$
\Delta T=\frac{3.4[\mathrm{ps}]}{0.65} \times 0.5 \simeq 2.62[\mathrm{ps}]
$$

for the TE polarization. Similarly, for the TM polarization, we have

$$
\Delta T=2.15[\mathrm{ps}] .
$$

Fig. 11 shows the relationship between the pulsewidth and the bandwidth assuming the exponential and the sech ${ }^{2}$ output pulse shapes. In this figure, the measured values of the output pulse are also shown, by $\boldsymbol{\square}$ and $\bullet$ for the exponential pulse and by $\square$ and $\circ$ for the sech pulse shape. From Fig. 11, it can be seen that $\Delta T \cdot \Delta \nu$ of the actual output pulse has a value between the exponential pulse and the sech pulse. Thus, the actual output pulse has a shape in between the exponential function, which has broad pulse wings and the sech function which has lower wings.

\section{CONCLUSION}

We measured the ultrashort pulse transmission characteristics of a VCMRR filter. It was confirmed that the maximum transmission capacity per channel of the filter with FWHM of 0.75 $\mathrm{nm}(94 \mathrm{GHz})$ was approximately $50 \mathrm{~Gb} / \mathrm{s}$. The pulse broadening through the filter was theoretically analyzed using the FT, and it was shown that the pulsewidth can be accurately calculated from the spectrum response of the filter. Although the maximum transmission capacity was confirmed to be about $50 \mathrm{~Gb} / \mathrm{s}$, the channel spacing is not determined by the bandwidth of the filter but by the large wings of Lorentzian response. This problem can be improved by incorporating higher order resonators [1] and cascaded microring resonators [7].

\section{REFERENCES}

[1] B. E. Little, S. T. Chu, H. A. Haus, J. Foresi, and J. P. Laine, "Microring resonator channel dropping filters," J. Lightwave Technol., vol. 15, pp. 998-1005, June 1997.

[2] C. K. Madsen, G. Lents, A. J. Bruce, M. A. Capuzzo, L. T. Gomez, T. N. Nielsen, and I. Brener, "Multistage dispersion compensator using ring resonators," Opt. Lett., vol. 24, pp. 1555-1557, Nov. 1999.

[3] B. E. Little, J. S. Foresi, H. A. Haus, E. P. Ippen, W. Greene, and S. T. Chu, "Ultra-compact $\mathrm{Si} / \mathrm{SiO}_{2}$ micro-ring resonator channel dropping filter," IEEE Photon. Technol. Lett., vol. 10, pp. 549-551, Aug. 1998.

[4] S. Suzuki, K. Shuto, and Y. Hibino, "Integrated-optic ring resonators with two stacked layers of silica waveguide on Si," IEEE Photon. Technol. Lett., vol. 4, pp. 1256-1258, Nov. 1992.

[5] B. E. Little, S. T. Chu, W. Pan, D. Ripin, T. Kaneko, Y. Kokubun, and E. Ippen, "Vertically coupled glass microring resonator channel dropping filters," IEEE Photon. Technol. Lett., vol. 11, pp. 215-217, Feb. 1999.

[6] S. T. Chu, B. E. Little, W. Pan, T. Kaneko, S. Sato, and Y. Kokubun, "An eight-channel add-drop filter using vertically coupled microring resonators over a cross grid," IEEE Photon. Technol. Lett., vol. 11, pp. 691-693, June 1999.

[7] S. T. Chu, B. E. Little, W. Pan, T. Kaneko, and Y. Kokubun, "Cascaded microring resonators for crosstalk reduction and spectrum cleanup in Add-Drop filters," IEEE Photon. Technol. Lett., vol. 11, pp. 1423-1425, Nov. 1999.

[8] S. T. Chu, B. E. Little, W. Pan, T. Kaneko, and Y. Kokubun, "Secondorder filter response from parallel coupled glass microring resonators," IEEE Photon. Technol. Lett., vol. 11, pp. 1426-1428, Nov. 1999.

[9] S. T. Chu, W. Pan, S. Sato, T. Kaneko, B. E. Little, and Y. Kokubun, "Wavelength trimming of a microring resonator filter by means of a UV sensitive polymer overlay," IEEE Photon. Technol. Lett., vol. 11, pp. 688-690, June 1999.

[10] S. T. Chu, W. Pan, S. Suzuki, B. E. Little, S. Sato, and Y. Kokubun, “Temperature insensitive vertically coupled microring resonator Add-Drop filters by means of a polymer overlay," IEEE Photon. Technol. Lett., vol. 11, pp. 1138-1140, Sept. 1999.

[11] G. Lents, B. J. Eggleton, C. K. Madsen, C. R. Giles, and G. Nykolak, "Optimal dispersion of optical filters for WDM systems," IEEE Photon. Technol. Lett., vol. 10, pp. 567-569, Apr. 1998.

[12] G. Lents, B. J. Eggleton, C. R. Giles, C. K. Madsen, and R. E. Slusher, "Dispersive properties of optical filters for WDM systems," J. Quantum Electron., vol. 34, pp. 1390-1402, Aug. 1998.

[13] M. Nakazawa, E. Yoshida, and Y. Kimura, "Ultrastable harmonically and regeneratively modelocked polarization-maintaining erbium fiber laser," Electron. Lett., vol. 30, pp. 1603-1604, 1994.

Shuichi Suzuki was born in Kanagawa, Japan, on October 6, 1976. He received the B.E. degree in electrical and computer engineering in 1999 from Yokohama National University, Yokohama, where he is currently pursuing the M.E. degree. His main interests include waveguide-type functional devices.

Mr. Suzuki is a member of the Institute of Electronics, Information, and Communication Engineers (IEICE) of Japan.

Yasuo Kokobun (M'85) was born in Fukushima, Japan, on July 7, 1952. He received the B.E. degree from Yokohama National University, Yokohama, Japan, in 1975 and the M.E. and Dr. Eng. degrees from Tokyo Institute of Technology, Tokyo, Japan, in 1977 and 1980, respectively.

From 1980 to 1983, he was a Research Associate at the Research Laboratory of Precision Machinery and Electronics, Tokyo Institute of Technology. In 1983, he joined the Yokohama National University as an Associate Professor, where he is now a Professor in the Department of Electrical and Computer Engineering. His current research is in integrated photonics, especially waveguide-type functional devices. From 1984 to 1985, he was with AT\&T Bell Laboratories, Holmdel, NJ, as a Visiting Researcher and was engaged in a novel waveguide on semiconductor substrate (ARROW) for integrated optics.

Dr. Kokubun is a member of the Japan Society of Applied Physics and the Optical Society of America (OSA). 
Masataka Nakazawa (M'84-SM'92-F'95) was born on September 17, 1952 , in Yamanashi, Japan. He received the B.S. degree from Kanazawa University, Japan, in 1975 and the M.S. and Ph.D. degrees from the Tokyo Institute of Technology, Toyko, Japan, in 1977 and 1980, respectively.

In 1980, he joined the Ibaraki Electrical Communication Laboratory of Nippon Telegraph \& Telephone Public Corporation (NTT), where he has been engaged in research on the transmission characteristics of optical fibers. He was with the Picosecond Optics and Quantum Electronics Group of the Massachusetts Institute of Technology (MIT), Cambridge, as a Visiting Scientist from 1984 to 1985. Since 1989, he has been the Group Leader of the High-Speed Nonlinear Optical Transmission Research Group at NTT Laboratories, Yokosuka, Japan, and became an NTT R\&D Fellow in 1999. He has also been a Visiting Professor of Tohoku University since 1998. He is the author or coauthor of over 290 journal articles and holds more than 50 patents. His current interests are optical soliton and ultra-high-speed transmission, femtosecond pulse generation, fiber amplifiers and their applications.

Dr. Nakazawa received the Niwa Memorial Award in 1980, the Best Paper Award for Optics from the Japan Society of Applied Physics in 1984, the Sakurai Memorial Award in 1989 from the OITDA, the Electronics Letters Premium Award in 1990 from the Institute of the Electrical Engineers, the Outstanding Achievement Award of IEICE in 1994 and 1996, the Minister Award of the Science and Technology Agency in 1997, as well as nine other awards. He is a Fellow of the Optical Society of America (OSA) and a member of the Japan Society of Applied Physics and the Laser Society of Japan.
Takashi Yamamoto was born in Nagasaki, Japan, in 1967. He received the B.E. and M.E. degrees in physics from Kyushu University, Fukuoka, Japan, in 1989 and 1991, respectively.

In 1991, he joined the Nippon Telegraph \& Telephone (NTT) Laboratories, Yokosuka, Japan, where he has been engaged in research on nonlinear fiber optics and ultrafast OTDM transmission.

Mr. Yamamoto is a member of the Institute of Electronics, Information, and Communication Engineers (IEICE) of Japan and the Japan Society of Applied Physics.

Sai Tak Chu (M'90), photograph and biography not available at the time of publication. 